\title{
Comparative Analysis of G.M Tube Characteristics Using (Ba-133) Gamma Source and (Sr-90) Beta Test Source
}

\author{
Aondoakaa J.K \\ Nigerian Nuclear Regulatory Authority Plot 564/565 \\ Airport Road, P.M.B 589, Abuja
}

\author{
Iortile J.T \\ Department of Radiology, BSUTH, \\ P.M.B 202131, Makurdi
}

\section{ABSTRACT}

The study is a recap of experimental physics which compares the G.M tube characteristics of (Ba-133) and (Sr-90) source respectively. SPECTECH ST360 Counter was used for this experiment. The test sources where placed $2 \mathrm{~cm}$ and $4 \mathrm{~cm}$ respectively in the counter window and voltage applied through starting at a potential of $780 \mathrm{v}$. The counter was allowed to read for $60 \mathrm{sec}$ as the voltages was increased in step of 20v. Results obtained using Microsoft mathematics and further evaluations showed an effective performance of $22.16 \%$ and $3.91 \%$ respectively for both the gamma and beta source.

Keywords: G.M tube Characteristics, Gamma and Beta Test Source.

\subsection{Introduction:}

A Geiger-Müller (GM) tube is a gas-filled radiation detector. It commonly takes the form of a cylindrical outer shell (cathode) and the sealed gas-filled space with a thin central wire (the anode) held at $\sim 1 \mathrm{KV}$ positive voltage with respect to the cathode. ${ }^{[1][2]}$ The fill gas is inert, generally argon at a pressure of less than 0.1 atm with a small quantity ofa quenching vapour $^{[3]}$.A typical Geiger-Müller (GM) Counter consists of a GM tube having a thin end-window made primarily of mica, a high voltage supply for the tube, a scaler to record the number of particles detected by the tube, and a timer which will stop the action of the scaler at the end of a pre-set interval ${ }^{[3]}$.
If a gamma ray ( $\gamma$ - source) interacts with the GM tube (primarily with the wall by either the Photoelectric Effect or Compton scattering) it will produce an energetic electron that may pass through the interior of the tube.

Gases conduct electricity only when a number of their atoms are ionized, i.e. split up into a number of free electrons and positive ions. Alpha, Beta or Gamma radiation emitted by radioactive materials ionizes atoms with which they collide. Geiger and Müller (1928), associates of Rutherford used this property to invent a sensitive detector for radiation named Geiger Müller Counter (G.M Counter). The techniques used in these measurements depend on the fact that the radioactive isotopes emit ionizing radiations, which can be detected by their effects on a photographic emulsion, or by electrical methods. The sensitivity of the GM tube is such that any particle capable of ionizing a single atom of the filling gas of the tube will initiate an avalanche of electrons and ions in the tube. The collection of the charge thus produced results in the formation of a pulse of 300-500 $\mu \mathrm{sec}$ voltage ${ }^{[4]}$ at the output of the tube. The amplitude of this pulse, on the order of volts, is sufficient to operate the scalar circuit with little or no further amplification. The pulse amplitude is largely independent of the properties of the particle detected, and gives therefore little information as to the nature of the particle.

Barium -133 (Ba-133) is the most stable of all the 33 isotopes of Barium; it has a half-life of 10.5 years, and emits a whole range of gamma radiation. Strontium90 (Sr-90) is a radioactive isotope of strontium 
produced by nuclear fission, with a half-life of 28.8 years. It undergoes $\beta$ - decay into Yttrium-90 (Y-90) which makes up about $5 \%$ of the nuclear daughter isotopes when Uranium is fissioned, with decay energy of $0.546 \mathrm{MeV} .^{[5][6]}$

Though the G.M tube does not conventionally distinguish different types of radiation but the research establishes a fact that the tube characteristics can serve as a parameter for differentiating the various types of radiation. The study compares the tube characteristics of Gamma and Beta radiation using (Ba-133) and (Sr-90) test source respectively.

\subsection{Theory:}

When ionizing radiation enters the tube, some of the molecules of the filled gas are ionized by the incident radiation forming ion-electron pair called primary ionization and these moves to the electrodes. The strong electric field created by the tube's electrodes accelerates the positive ions towards the cathode and the electrons towards the anode.

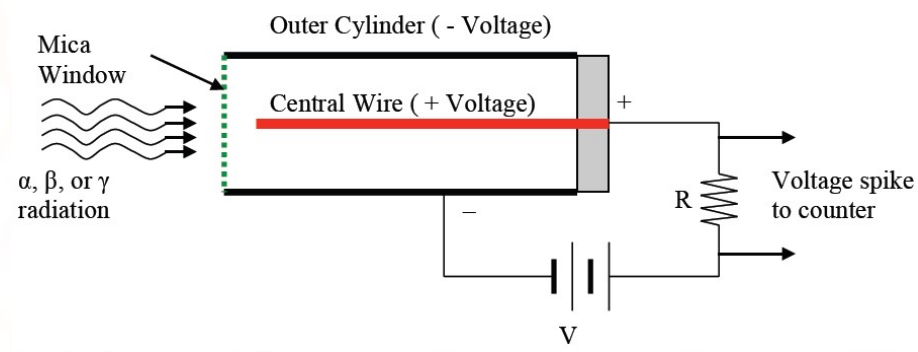

Fig1: Schematic Diagram of a typical G.M Counter

If the potential difference $(\mathrm{V})$ is increased further, the electron produced in the gas gain sufficient energy to ionize additional gas molecules, creating a large number of electron avalanche which spread along the anode. This is called secondary ionization, the electron average cover the entire length of the wire producing current pulses known as gas amplification. The ratio of electron reaching the anode (secondary ionization) to the number of electron formed initially in the gas (primary ionization) is a constant for a given voltage and the gas pressure. This is known as gas amplification factor given in the order of $10^{6}$ $10^{8}$. ${ }^{[3]}$

The avalanche electron is inversely proportional to the number of ion electron pairs initially formed by the incident radiation in the gas. This means that low and high energy rays will give rise to the same amplitude of the pulse. Therefore, the G.M Counter does not measure the energy of radiation. However, there is a voltage region within which changes of applied voltage to the counter does not affect changes in measured number of counts by the detector. This is called Geiger Plateau shown in fig2 below. The Geiger Plateau is the voltage range in which the Geiger Counter operates depending on the characteristics of the specific tube (e.g. manufacturer, size, gas type etc.) the exact voltage range may vary. ${ }^{[7]}$

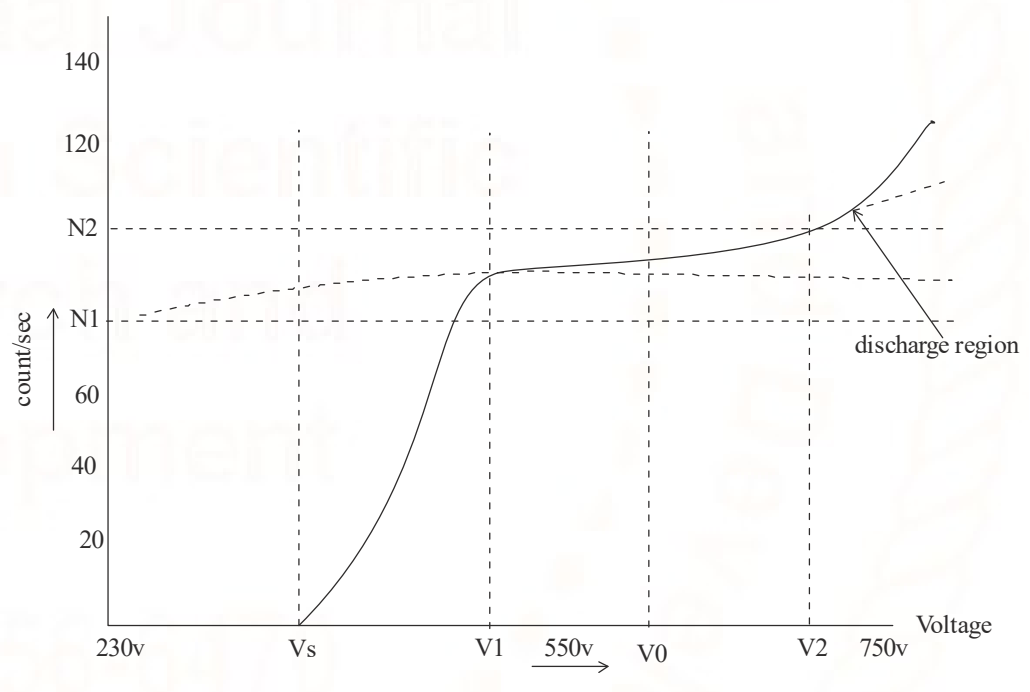

Fig2: G.M Characteristics Graph

The plateau region has a right upward slope given by equation 1.1

$\frac{\mathrm{N} 2-\mathrm{N} 1}{\mathrm{~N} 1} \cdot \frac{100}{\mathrm{~V} 2-\mathrm{V} 1} \times 100$

Where $\mathrm{N}_{1}$ and $\mathrm{N}_{2}$ are the count rates at lower and upper limits of the plateau and $\mathrm{V}_{1}$ and $\mathrm{V}_{2}$ are the corresponding voltages. A slope of $10 \%$ is desirable for effective performance. 
The operatingvoltage $\left(\mathrm{V}_{\mathrm{OP}}\right)$ is in the middle of the Geiger plateau. Theoretically this is given by the operating voltage shown in equation 1.2 below $^{[8]}$.

$$
(\mathrm{Vop})=\frac{\mathrm{V} 2+\mathrm{V} 1}{2}
$$

Where $\mathrm{V}_{1}$ is the lower threshold voltage and $\mathrm{V}_{2}$ is the upper threshold voltage.

\subsection{Experimental Set up and Procedure:}

The SPECTECH ST360 Counter, Barium (Ba-133) Gamma test source and Strontium (Sr-90) Beta test source where used for this research. A connection from the G.M tube to the G.M connector was made using a BNC cable. Details of the setup are contained in the Physics practical manual Benue State University. The mains cord was also connected from the counting system to $230 \mathrm{~V}$ A.C power.

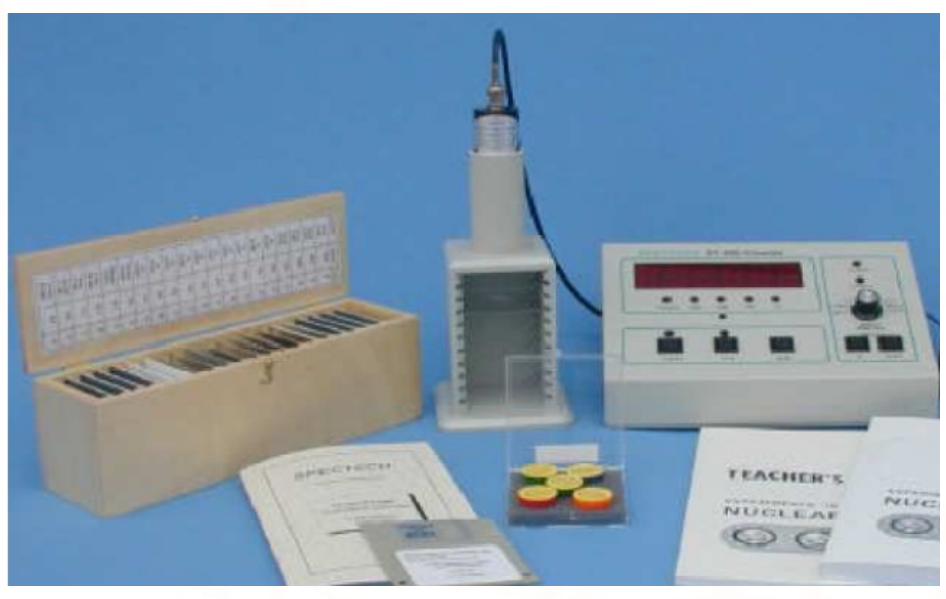

Fig 3: G.M Counter Setup

The gamma source (Ba-133) was placed facing the end window of the detector in the source holder of the
G.M Counter stand (optical bench) at $2 \mathrm{~cm}$ distance and $4 \mathrm{~cm}$ for the beta source (Sr-90). The counting system was set to $60 \mathrm{sec}$ on the control console; and the counter set in counting mode to record the counts for every step of $20 \mathrm{~V}$ for the pre-set time of $60 \mathrm{sec}$. Readings were taken with the source and without the source for background counts.

A plot of corrected counts against voltage was done using Microsoft Mathematics software (Mathematical). The plateau region was determined using equation 1.3 below.

$\frac{\mathrm{N} 2-\mathrm{N} 1}{\mathrm{~N} 1} \cdot \frac{100}{\mathrm{~V} 2-\mathrm{V} 1} \times 100$

Where $\mathrm{N}_{1}$ and $\mathrm{N}_{2}$ are the count rates at lower and upper limits of the plateau and $\mathrm{V}_{1}$ and $\mathrm{V}_{2}$ are the corresponding voltages.

The plateau potential length $\left(\mathrm{V}_{\mathrm{PL}}\right)$ is the difference between the upper and lower potential i.e.

$\mathrm{V}_{\mathrm{PL}}=\mathrm{V}_{2}-\mathrm{V}_{1}$

The operating voltage $(\mathrm{Vo})=\frac{\mathrm{V} 2+\mathrm{V} 1}{2}$

\subsection{Results:}

Results obtained from the G.M Counter are presented in table 1.0 and table 2.0 for the gamma and beta source respectively. Microsoft Mathematics software was used to obtain the G.M tube characteristics graph for both the gamma and beta source. This is shown in fig 3 and 4 respectively. 
International Journal of Trend in Scientific Research and Development, Volume 1(4), ISSN: 2456-6470 www.ijtsrd.com

Table 1.0 Gamma Source (Ba-133)

\begin{tabular}{cccccc}
\hline S/No & EHV(V) & $\begin{array}{c}\text { No of counts } \\
\text { 60s }\left(\mathbf{N}_{\mathbf{1}}\right)\end{array}$ & $\begin{array}{c}\text { Background } \\
\text { Count 60s } \\
\left.\mathbf{( N}_{\mathbf{0}}\right)\end{array}$ & $\begin{array}{c}\text { Corrected } \\
\text { Counts (sec) } \\
\mathbf{N c}=\left(\mathbf{N}_{\mathbf{1}}-\mathbf{N}_{\mathbf{o}}\right)\end{array}$ & $\begin{array}{c}\text { Count rate } \\
(\mathbf{s e c})\end{array}$ \\
\hline $\mathbf{1}$ & 780 & 114 & 5 & 109 & 0 \\
\hline $\mathbf{2}$ & 800 & 556 & 22 & 534 & 15 \\
\hline $\mathbf{3}$ & 820 & 636 & 20 & 616 & 12 \\
$\mathbf{4}$ & 840 & 666 & 26 & 640 & 6 \\
$\mathbf{5}$ & 860 & 674 & 30 & 644 & 9 \\
$\mathbf{6}$ & 880 & 693 & 26 & 667 & 11 \\
$\mathbf{7}$ & 900 & 690 & 23 & 667 & 10 \\
$\mathbf{8}$ & 920 & 680 & 27 & 653 & 8 \\
\hline $\mathbf{9}$ & 940 & 700 & 24 & 676 & 16 \\
\hline $\mathbf{1 0}$ & 960 & 855 & 31 & 824 & 11 \\
\hline
\end{tabular}

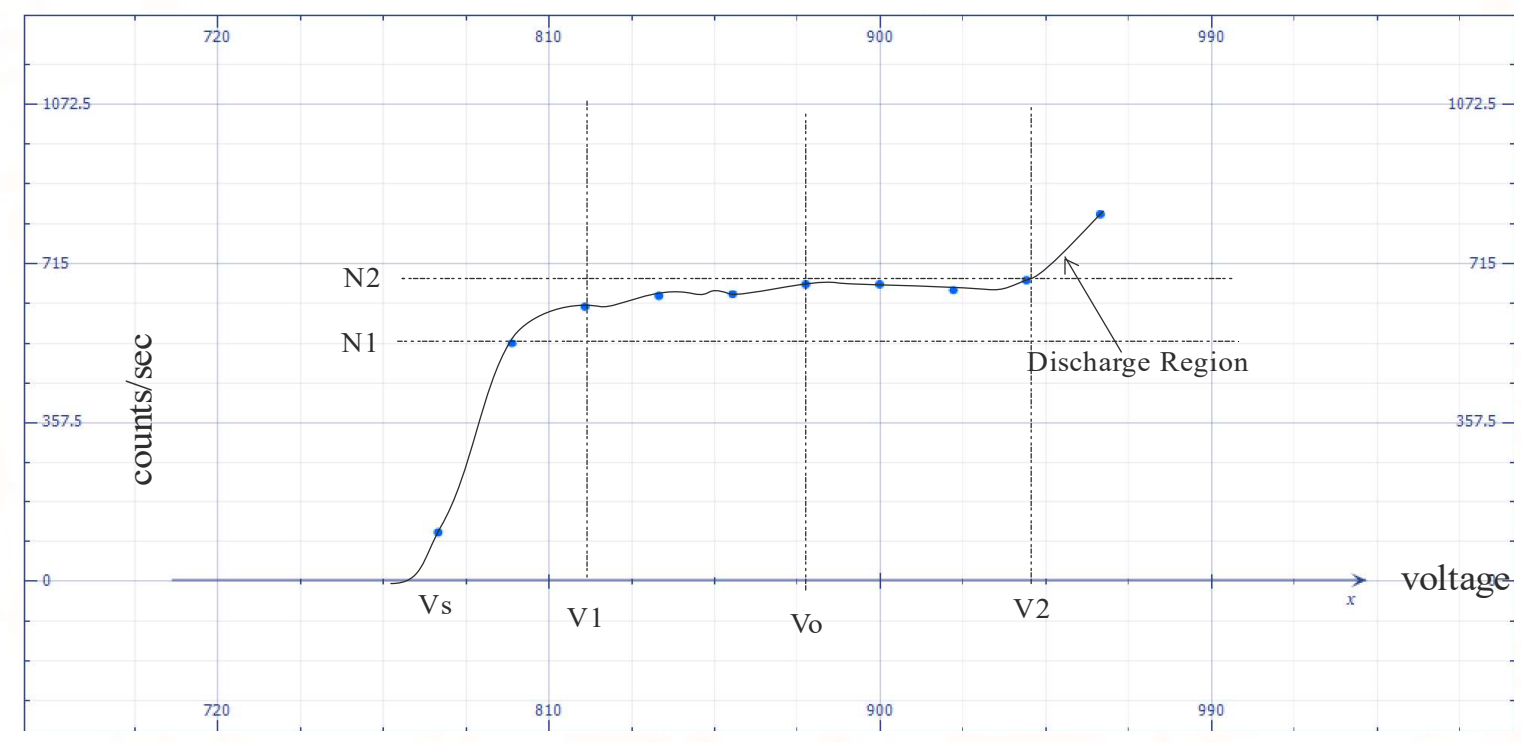

Fig 4: G.M Tube Characteristics graph of (Ba-133) Gamma Source 
International Journal of Trend in Scientific Research and Development, Volume 1(4), ISSN: 2456-6470 www.ijtsrd.com

Table 2.0: Beta Source (Sr-90)

\begin{tabular}{|l|l|l|l|l|l|}
\hline S/No & EHV(V) & $\begin{array}{l}\text { No of counts } \\
\mathbf{6 0 s}\left(\mathbf{N}_{\mathbf{1}}\right)\end{array}$ & $\begin{array}{l}\text { Background } \\
\left.\text { Count 60s } \mathbf{( N}_{\mathbf{0}}\right)\end{array}$ & $\begin{array}{l}\text { Corrected } \\
\text { Counts } \\
\left.\mathbf{N c}=\mathbf{( N}_{\mathbf{1}}-\mathbf{N}_{\mathbf{0}}\right)\end{array}$ & $\begin{array}{l}\text { Count rate } \\
(\mathbf{s e c})\end{array}$ \\
\hline $\mathbf{1}$ & 780 & 29 & 1 & 28 & 1 \\
\hline $\mathbf{2}$ & 800 & 1885 & 20 & 1865 & 28 \\
\hline $\mathbf{3}$ & 820 & 1916 & 31 & 1909 & 41 \\
\hline $\mathbf{4}$ & 840 & 1940 & 16 & 1924 & 39 \\
\hline $\mathbf{5}$ & 860 & 2090 & 14 & 2076 & 35 \\
\hline $\mathbf{6}$ & 880 & 1997 & 18 & 1979 & 30 \\
\hline $\mathbf{7}$ & 900 & 2038 & 17 & 2021 & 30 \\
\hline $\mathbf{8}$ & 920 & 2021 & 21 & 2000 & 39 \\
\hline $\mathbf{9}$ & 940 & 2060 & 20 & 2040 & 34 \\
\hline $\mathbf{1 0}$ & 960 & 2269 & 14 & 2255 & 31 \\
\hline
\end{tabular}

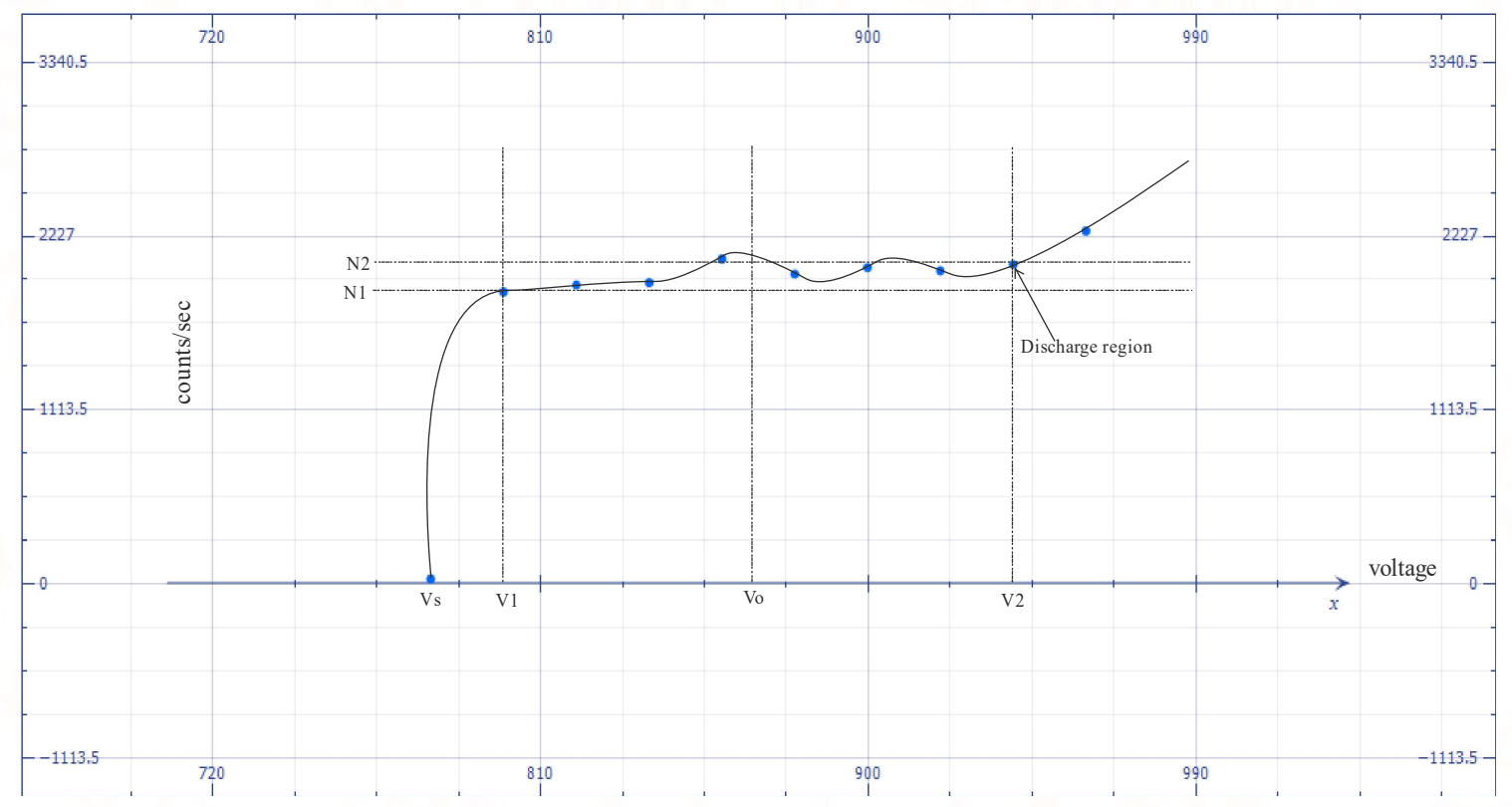

Fig 5: G.M Tube Characteristics graph of (Sr-90) Beta Source

Other deductions from equations 1.3, 1.4 and 1.5 are presented in table 3 below 
Table 3.0: Other Evaluated Parameters

\begin{tabular}{|c|c|c|c|}
\hline S/No & Parameter & (Ba-133) & (Sr-90) \\
\hline $\mathbf{1 .}$ & $\mathrm{V}_{\mathrm{o}(\mathrm{V})}$ & 880 & 870 \\
\hline $\mathbf{2 .}$ & $\mathrm{V}_{1(\mathrm{~V})}$ & 820 & 800 \\
\hline $\mathbf{3 .}$ & $\mathrm{V}_{2(\mathrm{~V})}$ & 940 & 940 \\
\hline $\mathbf{4 .}$ & $\mathrm{V}_{\mathrm{S}(\mathrm{V})}$ & 780 & 780 \\
\hline $\mathbf{5 .}$ & $\mathrm{V}_{\mathrm{PL}(\mathrm{V})}$ & 120 & 140 \\
\hline $\mathbf{6 .}$ & $\mathrm{N}_{1(\mathrm{~S})}$ & 534 & 1865 \\
\hline $\mathbf{7 .}$ & $\mathrm{N}_{2(\mathrm{~S})}$ & 676 & 2040 \\
\hline $\mathbf{8 .}$ & Plateau Region $(\%)$ & 22.16 & 3.91 \\
\hline
\end{tabular}

\subsection{Result Analysis:}

Table 1 and 2 shows the measured values of counts using 780 volts as starting voltage for both gamma and beta source. This is because 780 volts was the potential at which the tube began to detect significant radiation and displayed result. Fig4 is the G.M tube characteristics of the gamma radiation source. It is a curved graph of count per second against voltage. The graph shows that at 780 volts the counter starts to detect some level of ionization and as the potential increases, the number of counts also increases steadily till at a particular potential of $940 \mathrm{v}$ where changes in further application of voltage does not affect changes in the measured number of counts by the detector. This region is called the Geiger Plateau. In Fig5, the Geiger plateau is observed at the same potential of $940 \mathrm{v}$ for the beta source as well.

The lower and upper threshold voltage for both the gamma and beta source is presented in table 3 above. The slope of the plateau region for both the gamma and beta source, which was calculated using equation
1.3 , gave the values $22.6 \%$ and $3.91 \%$ respectively. This implies that Sr-90 beta source is more efficient since a slope of less than $10 \%$ is desirable for effective performance.

Generally, the measured values of the Sr-90 beta source are observed to be higher compared to those of Ba-133 gamma source, and also as observed in the shape of the characteristics curve for the gamma and beta source given in fig4 and fig5 respectively. Thus these features can be used as bases for differentiating different types of radiation when a Geiger counter is used to detect radiation for experimental purposes.

\subsection{Conclusion:}

G.M tube is well known to detect all types of radiation; it is a common instrument easily available all across the world for industrial and research purposes.The comparative analysis of the tube characteristics using Ba-133 gamma test source and Sr-90 beta test source has shown explicitly the working mechanism of the instrument for 
experimental purpose. Based on the measured values

it is evident that beta radiation has higher strength compared to gammas radiation. The study also shows a plateau region of less than $10 \%$ for beta radiation which shows the G.M tube effective performance when a beta source is used. Again with the distinguishing features evident in the characteristic curves, the G.M tube characteristics experiment serves as a parameter for differentiating different types of radiation when a G.M counter is used to detect radiation.

\section{Acknowledgment}

All thanks to God for the gift of knowledge and understanding. With great joy the authors are grateful to the Chief Technologist Department of Physics Benue State University Makurdi for his technical assistance. Appreciation to Dr. Aondoakaa Kwaghfan for supportive in sponsorship and otherwise, not forgetting also Mr Usman Ismaila of the Nigerian Nuclear and Regulatory Authority for his encouragement. God bless you all.
Reference:

[1]. Geiger, H.; Müller, W. (1928). "Elektronenzählrohr zur Messung schwächster Aktivitäten" [Electron counting tube for measurement of weakest radioactivities]. Die Naturwissenschaften (in German). 16 (31): 617-618.

[2]. N. Tsoulfanidis, Measurement and Detection of Radiation, (McGraw Hill Book Company, 1983).

[3]. Glenn F Knoll. Radiation Detection and Measurement, third edition 2000. John Wiley and sons, ISBN 0-471-07338-5

4.

www.physics.ryerson.ca/sites/default/files/u11/exp/.../ TheGeigerCounter.pdf

[5]. "Strontium | Radiation Protection | US EPA". EPA. 24 April 2012. Retrieved 18 June 2012.

[6]. "Table of Isotopes decay data". Lund University. Retrieved 2014-10-13 reference about strontium.

[7]. National Council on Radiation Protection and Measurements (NCRP, 1985). A Handbook of Radioactivity Measurements Procedures (2nd ed.) pp. 30-31. ISBN 0-913392-71-5. Report No. 58.

[8]. Physics Practical Manual: Department of Physics Benue State University. Reviewed 2015; Pp. (63-65) 